\title{
Ocorrência de Thaumastocoris peregrinus Carpintero \& Dellapé (Hemiptera: Thaumastocoridae) no Estado de Goiás
}

\author{
Occurence of the Thaumastocoris peregrinus Carpintero \& Dellapé (Hemiptera: Thaumastocoridae) in \\ Goiás State
}

\author{
Jaqueline Magalhães Pereira ${ }^{I^{*}}$ Aniela Pilar Campos de Melo ${ }^{\mathrm{I}}$ Paulo Marçal Fernandes ${ }^{\mathrm{I}}$ \\ Everton Pires Soliman ${ }^{\text {II }}$
}

- NOTA -

\section{RESUMO}

O percevejo bronzeado Thaumastocoris peregrinus Carpintero \& Dellapé (Hemiptera: Thaumastocoridae) foi encontrado no Estado de Goiás atacando plantas de Eucalyptus camaldulensis Dehnh. e E. urophylla S. T. Blake. A detecção ocorreu nos municípios de São Luiz do Norte, São Francisco de Goiás e Goiânia.

Palavras-chave: percevejo bronzeado, praga florestal, Eucalyptus.

\section{ABSTRACT}

The bronze bug Thaumastocoris peregrinus Carpintero \& Dellapé (Hemiptera: Thaumastocoridae) was found in Goiás state attacking Eucalyptus camaldulensis Dehnh. e E. urophylla S. T. Blake. The insect was detected in the cities of São Luiz do Norte, São Francisco de Goiás and Goiânia.

Key words: bronze bug, forestry pest, Eucalyptus.

O percevejo bronzeado do eucalipto Thaumastocoris peregrinus Carpintero \& Dellapé 2006 (Hemiptera: Thaumastocoridae), nativo da Austrália, foi detectado no Brasil em 2008 em São Francisco de Assis, RS (WILCKEN et al., 2010). Esse inseto se disseminou rapidamente nos principais estados produtores. Sua presença foi confirmada em São Paulo, Minas Gerais, Espírito Santo, Rio de Janeiro, Mato Grosso do Sul (WILCKEN et al., 2010), Paraná
(BARBOSA et al., 2010) e Santa Catarina (SAVARIS et al., 2011). O percevejo bronzeado já foi relatado em outros países como a África do Sul (JACOBS \& NESER, 2005), Zimbábue, Malaui (NADEL et al., 2010), Argentina (NOACK \& COVIELLA, 2006), Uruguai (MARTÍNEZ \& BIANCHI, 2010), Quênia (NOACK et al., 2011), Chile (IDE et al., 2011), Itália (LAUDONIA \& SASSO, 2012) e Nova Zelândia (SOPOW et al., 2012).

Os adultos de $T$. peregrinus são pequenos (2 a $3 \mathrm{~mm}$ de comprimento), corpo achatado e coloração marrom. Estes são caracterizados por placas mandibulares recurvadas, apêndices apicais nas tíbias e cápsula genital masculina assimétrica predominantemente orientada para a direita (NOACK et al., 2011). Os ovos são pretos e geralmente encontramse agrupados em irregularidades de folhas e ramos (JACOBS \& NESER, 2005). A fase ninfal possui cinco ínstares (NOACK \& ROSE, 2007). Os machos apresentam comportamento de agregação que está associado à presença de feromônio (GONZÁLEZ et al., 2012). No entanto, este semioquímico também é produzido por fêmeas, em pequenas quantidades, sugerindo que este composto não está associado à agregação para o acasalamento (MARTINS et al., 2012). Os adultos e as ninfas ocasionam danos devido à sucção de seiva. Os danos estão relacionados à queda de folhas acarretando a diminuição da área

\footnotetext{
'Universidade Federal de Goiás (UFG), Escola de Agronomia e Engenharia de Alimentos, CP 131, 74690-900, Goiânia, GO, Brasil. E-mail: jaqueline@agro.ufg.br. *Autor para correspondência.

"Universidade Estadual Paulista Júlio de Mesquita Filho (UNESP), Faculdade de Ciências Agronômicas, Botucatu, São Paulo, SP, Brasil.
} 
fotossintética; e em altas populações, até a morte das plantas. Em árvores infestadas inicialmente é observado um prateamento das folhas, seguido por um bronzeamento, devido a esta característica é denominado vulgarmente de percevejo bronzeado (JACOBS \& NESER, 2005; WILCKEN et al., 2010).

Desta forma, este estudo relata a ocorrência de $\boldsymbol{T}$. peregrinus atacando plantas de Eucalyptus camaldulensis Dehnh. e E. urophylla S. T. Blake no Estado de Goiás.

As observações de $\boldsymbol{T}$. peregrinus foram realizadas em novembro de 2011 em árvores isoladas de $\boldsymbol{E}$. camaldulensis, no município de São Luiz do Norte (15 55'29” S; 49 15'28' O; 578m altitude - próximas a rodovia BR-153). Posteriormente, a presença do percevejo foi verificada nos municípios de São Francisco de Goiás (14 51' 15" S; 49 19'9” O; 750m altitude - próximas a rodovia GO-080) em árvores de $\boldsymbol{E}$. camaldulensis, e Goiânia (16³2’39” S; 49 15'57' O; $760 \mathrm{~m}$ altitude - próximas a rodovia GO-462) em árvores de $\boldsymbol{E}$. urophylla. Aleatoriamente foi observada a presença do inseto em ramos e folhas. Os exemplares foram coletados e fixados em álcool $70 \%$ e encaminhados para o laboratório para análise. A identificação dos espécimes de $\boldsymbol{T}$. peregrinus foi realizado por meio de ilustrações e caracterização descrita por CARPINTERO \& DELLAPÉ (2006) e WILCKEN et al. (2010).

Nessas árvores foram observados ovos, ninfas e adultos do percevejo bronzeado em baixa população (Figura 1). As folhas apresentavam prateamento, porém, não foram observadas desfolha e coloração bronzeada, devido à quantidade de insetos presentes nas plantas. A menor infestação encontrada pode estar relacionada a condições climáticas desfavoráveis durante o período de detecção, principalmente devido à ocorrência de chuvas. Porém, existem poucas informações sobre os fatores que afetam a distribuição e abundância do percevejo bronzeado (NADEL \& NOACK, 2012).

Thaumastocoris peregrinus tem se dispersado facilmente desde a sua introdução no país em 2008. É importante ressaltar que este possui elevada capacidade de adaptação, já que pode sobreviver em condições climáticas diferentes das demais regiões em que foi encontrado. O clima na região onde o percevejo foi encontrado em Goiás é do tipo Aw, caracterizado por inverno seco e verão chuvoso. A região sul possui o clima do tipo Cfa - clima subtropical com verão quente e $\mathrm{Cfb}$ - clima temperado com o verão ameno. Já na região sudeste verifica-se o clima Aw, Cfa, Cfb, Af (clima tropical úmido ou superúmido, sem estação seca), Am (clima tropical úmido ou subúmido), Cwa (clima subtropical de inverno seco) e Cwb (clima subtropical de altitude, com inverno seco e verão ameno). Portanto, nota-se que há diversidade de temperatura média e precipitação nos locais em que o inseto está presente no país.

O percevejo bronzeado foi relatado nos seguintes hospedeiros no Brasil: E. camaldulensis, $\boldsymbol{E}$. tereticornis, E. urophylla, E. viminalis, híbridos de

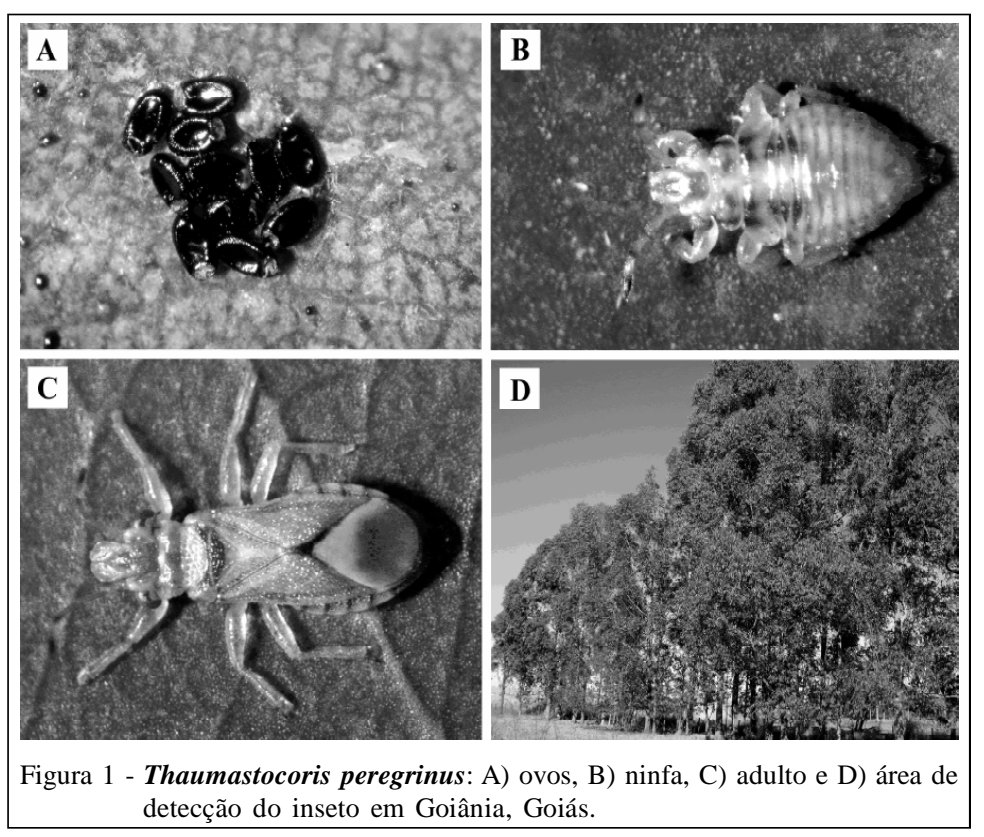

Ciência Rural, v.43, n.2, fev, 2013. 
E. grandis $\mathrm{x}$ E. urophylla, E. grandis $\mathrm{x} \boldsymbol{E}$. camaldulensis e E. urophylla $\times$ E. camaldulensis (WILCKEN et al., 2010). Além dessas, outras espécies são hospedeiras: $\boldsymbol{E}$. botryoides, $\boldsymbol{E}$. dorrigoensis, $\boldsymbol{E}$. nicholii, E. paniculata, E. punctata, E. saligna, $E$. scoparia, E. sideroxylon (JACOBS \& NESER, 2005), E. benthamii, E. globulus, E. bicostata e E. maidenii (NOACK \& COVIELLA, 2006).

O Estado de Goiás possui uma área aproximadamente 59 mil hectares de florestas de eucalipto (ABRAF, 2012), com possibilidade de expansão nos próximos anos. Desta forma, a presença do percevejo bronzeado no estado pode representar um risco para a cultura, já que as condições climáticas locais são semelhantes a outras regiões em que o inseto está presente e atingiu altas densidades populacionais. Além disso, a presença de espécies hospedeiras nos plantios comerciais, principalmente híbridos de $\boldsymbol{E}$. urophylla $\times$ E. grandis 'urograndis', podem contribuir para este aumento populacional.

Em relação às alternativas de controle, existem poucas informações sobre estratégias eficientes no manejo deste inseto em áreas comerciais de eucalipto. Há registro do uso do inseticida sistêmico imidacloprid em áreas urbanas na Austrália, por meio de microinjeção no tronco da árvore (NOACK et al., 2009). Porém, essa medida é viável somente para pequenas áreas como árvores isoladas em meio urbano devido ao alto custo. A utilização de inimigos naturais é uma boa alternativa para o atendimento das exigências relativas ao processo de certificação florestal e aos menores impactos ao homem e ao ambiente. $\mathrm{O}$ parasitoide de ovos Cleurochoides noackae Lin \& Huber (Hymenoptera: Mymaridae), é considerado um agente promissor no controle (LIN et al., 2007). Neste contexto, há relatos de predadores como Supputius cincticeps Stal (Hemiptera: Pentatomidae) e Chyrsoperla externa Hagen (Neuropteta: Chrysopidae) predando o percevejo bronzeado (WILCKEN et al., 2010; SOUZA et al., 2012). Em relação ao controle microbiano, foi observada a ocorrência natural do fungo Zoophthora radicans causando infecção em ninfas e adultos (MASCARIN et al., 2012).

O percevejo bronzeado está se disseminando rapidamente e tem mostrado alto potencial de adaptação em diferentes regiões produtoras. Esta dispersão pode ser influenciada pelo transporte de madeira (WILCKEN et al., 2010) e mudas de eucalipto (BARBOSA et al., 2010). Assim, os danos e prejuízos causados pelo percevejo bronzeado ao eucalipto podem ser intensificados devido ao aumento populacional nas épocas mais favoráveis. Portanto, estudos sobre a bioecologia (SOLIMAN, 2012) e dinâmica populacional nas diferentes condições brasileiras auxiliarão no manejo integrado desta praga.

A detecção de T. peregrinus em Goiás mostra a capacidade de dispersão e adaptação deste inseto nas diferentes regiões produtoras de eucalipto no Brasil.

\section{AGRADECIMENTOS} utilizadas neste trabalho.

\section{REFERÊNCIAS}

ABRAF. Anuário Estatístico da ABRAF- Ano base 2011. Brasília, ABRAF. 2012, 145p. Acessado em 20 ago. 2012. Online. Disponível em: 〈http://www.abraflor.org.br/estatisticas.asp〉.

BARBOSA, L.R. et al. Registro de Thaumastocoris peregrinus (Hemiptera: Thaumastocoridae) no estado do Paraná. Pesquisa Florestal Brasileira, Colombo, v. 30, n. 61, p.7577, jan./abr. 2010.

CARPINTERO, D.L.; DELLAPÉ, P.M. A new species of Thaumastocoris Kirkaldt from Argentina (Heteroptera: Thaumastocoridae: Thaumatocorinae). Zootaxa, Auckland, n.1228, p.61-68, 2006.

GONZÁLEZ, A. et al. A male aggregation pheromone in the bronze bug, Thaumastocoris peregrinus (Thaumastocoridae). Psyche, Cairo, v.2012. 2012. Disponível em: <http:// dx.doi:10.1155/2012/868474>. Acesso em: 29 jun. 2012. doi: $10.1155 / 2012 / 868474$.

IDE, S.M. et al. Detección de Thaumastocoris peregrinus (Hemiptera: Thaumastocoridae) associado a Eucalyptus spp. em Chile. Bosque, Valdivia, v.32, n.3, p.309-313, 2011. Disponível em: <http://www.scielo.cl/pdf/bosque/v32n3/ art12.pdf $>$. Acesso em: 03 jun. 2012. doi: 10.4067/S071792002011000300012 .

JACOBS, D.H.; NESER, S. Thaumastocoris australicus Kirkaldy (Heteroptera: Thaumastocoridae): a new insect arrival in South Africa, damaging to Eucalyptus trees: research in action. South African Journal of Science, Pretoria, v. 101, n. 5, p.233236, 2005.

LAUDONIA, S.; SASSO, R. The bronze bug, Thaumastocris peregrines: a new insect recorded in Italy, damaging to Eucalyptus trees. Bulletin of Insectology, Bologna, v. 65, n. 1, p. 89-93, 2012. Disponível em: <http://www.bulletinofinsectology.org/ pdfarticles/vol65-2012-089-093laudonia.pdf >. Acesso em: 09 ago. 2012 .

LIN, N.Q. et al. The Australian genera of Mymaridae (Hymenoptera: Chaladoidea). Zootaxa, Auckland, n.1596, 111p. set. 2007. Disponível em: <http://www.mapress.com/ zootaxa/2007f/z01596p111f.pdf>. Acesso em: 10 abr. 2012.

MARTÍNEZ, G.; BIANCHI, M. Primer registro para Uruguay de la chinche del eucalipto, Thaumastocoris peregrinus Carpintero y Dellappé, 2006 (Hemipera; Thaumastocoridae). Agrociencia, Montevideo, v.14, n.1, p.15-18, 2010. Disponível em: 〈http://www.scielo.edu.uy/pdf/agro/v14n1/v14n1a03.pdf>. Acesso em: 22 mar. 2012. 
MARTINS, C.B.C. et al. Volatile chemicals of adults and nymphs of the Eucalyptus pest, Thaumastocoris peregrinus (Heteroptera: Thaumastocoridae). Psyche, Cairo, v. 2012. 2012. Disponível em: <http://www.hindawi.com/journals/ psyche/2012/275128/>. Acesso em: 09 ago. 2012.

MASCARIN, G.M. et al. Natural occurrence of Zoophthora radicans (Entomophthorales: Entomophthoraceae) on Thaumastocoris peregrinus (Heteroptera: Thaumastocoridae), an invasive pest recently found in Brazil. Journal of Invertebrate Pathology, New York, v.110, n.3, p.401-404, 2012. Disponível em: <http://dx.doi.org/10.1016/j.jip.2012.03.025>. Acesso em: 01 jul. 2012. doi: 10.1016/j.jip.2012.03.025.

NADEL, R.L. et al. DNA bar-coding reveals source and patterns of Thaumastocoris peregrinus invasions in South Africa and South America. Biological Invasions, Dordrecht, v.12, p.1067-1077, 2010. Disponível em: <http://dx.doi.org/ 10.1007/s10530-009-9524-2>. Acesso em: 24 abr. 2012. doi: $10.1007 / \mathrm{s} 10530-009-9524-2$.

NADEL, R.L.; NOACK, A.E. Current understanding of the biology of Thaumastocoris peregrines in the quest for a management strategy. International Journal of Pest Management, London, v.58, n.3, p.257-266, 2012. Disponível em: <http:// dx.doi.org/10.1080/09670874.2012.659228>. Acesso em: 10 ago. 2012. doi: 10.1080/09670874.2012.659228.

NOACK, A.E.; COVIELLA, C.E. Thaumastocoris australicus Kirkaldy (Hemiptera: Thaumastocoridae): first Record of this invasive pest of Eucalyptus in the Americas. General Applied Entomology, New South Wales, v.35, n.2, p.13-14, 2006.

NOACK, A.E.: ROSE, H. Life-history of Thaumastocoris peregrinus and Thaumastocoris $\mathrm{sp}$. in the laboratory with some observations on behavior. General and Applied Entomology, New South Wales, v.36, p.27-33, 2007.

NOACK, A.E. et al. Efficacy of imidacloprid in the control of Thaumastocoris peregrinus on Eucalyptus scoparia in Sydney. Arboriculture \& Urban Forestry, Champaign, v.35, n.4, p.192-196, 2009. Disponível em: <http://joa.isa-arbor.com/ request.asp? JournalID $=1 \&$ ArticleID $=3108 \&$ Type $=2>$. Acesso em: 01 jun. 2012.

NOACK, A. E. et al. Systematic revision of efficacy of imidacloprid in the control of Thaumastocoris Kirkaldy (Hemiptera: Heteroptera: Thaumastocoridae). Zootaxa, Auckland, n. 3121, p.1-60, 2011

SAVARIS, M. et al. Primeiro registro de Thaumastocoris peregrinus para o estado de Santa Catarina, e novas áreas de ocorrência para o Rio Grande do Sul, Brasil. Ciência Rural, Santa Maria, v.41, n 11, p.1874-1876, nov. 2011. Disponível em: 〈http://dx.doi.org/10.1590/S0103-84782011001100004〉. Acesso em: 12 jun. 2012. doi: 10.1590/S010384782011001100004

SOLIMAN, E.P. et al. Biology of Thaumastocoris peregrinus Carpintero \& Dellapé (Hemiptera: Thaumastocoridae) in different eucalyptus species and hybrids. Phytoparasitica, Tel Aviv, v.40, p.223-230, 2012. Disponível em:<http://dx.doi.org/ 10.1007/s12600-012-0226-4>. Acesso em: 02 jul. 2012. doi: http://dx.doi.org/10.1007/s12600-012-0226-4.

SOPOW, S. et al. Bronze bug, Thaumastocoris peregrinus: a new Eucalyptus pest in New Zealand. Surveillance, Wellington, v.39, p.43-46, 2012. Disponível em: <http:// www.sciquest.org.nz/node/77408>. Acesso em: 16 ago. 2012.

SOUZA, G. et al. First record of a native heteropteran preying on the introduced Eucalyptus pest, Thaumastocoris peregrines (Hemiptera: Thaumastocoridae) in Brazil. Florida Entomologist, Lutz, v.95, n.2, p.517-520. 2012. Disponível em: 〈http://journals.fcla.edu/flaent/article/view/79321/76665>. Acesso em: 17 ago. 2012.

WILCKEN, C.F. et al. Bronze bug Thaumastocoris peregrinus Carpintero and Dellapé (Hemiptera: Thaumastocoridae) on Eucalyptus in Brazil and its distribution. Journal of Plant Protection Research, Poznán, v.50, n.2, p.210-205, 2010. Disponível em: <http://www.plantprotection.pl/PDF/50(2)/ JPPR 50(2) 14 Wilcken.pdf $>$. Acesso em: 23 abr. 2012. 